von Hofsten \& Rosander (Eds.)

Progress in Brain Research, Vol. 164

ISSN 0079-6123

Copyright (C) 2007 Elsevier B.V. All rights reserved representations

Abstract: At around 4 months of age, infants predict the reappearance of temporary occluded objects. Younger infants have not demonstrated such an ability, but they still benefit from experience; decreasing their reactive saccade latencies over successive passages from the earliest age tested (7 weeks of age). We argue that prediction is not an all or none process that infants either lack or possess. Instead, the ability to predict the reappearance of an occluded object is dependent on numerous simultaneous factors, including the occlusion duration, the manner in which the object disappears, and the previous experiences with similar events. Furthermore, we claim that infants' understanding of how occluded objects move is based on prior experiences with similar events. Initially, infants extrapolate occluded object motion, because they have massive experience with such motion. But infants also have the ability to rapidly adjust to novel trajectories that violate their initial expectations. All of these findings support a constructivist view of infants object representations.

\section{Taking an action perspective on infant's object representations}

As we move around in the environment, objects constantly disappear and reappear from behind one another due to occlusion. Despite this, we as adults manage to maintain a uniform view of the world by compensating for object translations and by representing those objects that are temporarily out of sight. This enables us to predict future events and makes us ready to interact with the environment in a goal directed manner.

Organizing actions towards objects that are temporarily out of view poses specific problems to the perceptual-cognitive system. In order to effectively act towards the future reappearance of a moving object, we must represent that object and be able to estimate both where and when it will reappear. This knowledge is essential for our ability to smoothly carry out action plans despite the fact that objects go in and out of view. Developing stable object representations signify a major improvement of an infants' capability to interact with the environment.

The development of children's understanding of object permanence has been debated with vigour since it was first discussed by Piaget (1954). He considered the development of object permanence to be extremely important. With the establishment of object permanence the child goes from living in a fractionated world with no continuity to a world where objects have permanent existence and unique identity. He claimed that infants do not possess an adult-like ability to represent temporarily occluded objects as permanently existing objects until they understand the sequential displacements of a hidden object at the end of the second year of life. At the same time he noted that 
1 infants begin to show signs of object representation already during the stage of 'secondary circular reactions', that is, between 4 and 8 months of age but only within the same modality. At this age infants will briefly look for an object that has disappeared but they will not try to retrieve it. From around 12 months of age, infants retrieve hidden objects. If, however, the object is hidden at the same place several times and then hidden at a different place, the infants will reach for it at the previous hiding locations (A not B). It has also been reported that infants in this situation will look at the correct hiding place but reach for the previous one (Mareschal, 2000). Obviously, the relationship between object representation and action is relatively complex.

Piaget's object permanence task is confounded in one important respect. When the object is hidden, the child has to search for it. Failing to do so might reflect inability to represent the hidden object (out of sight - out of mind) but it might also be caused by inability to formulate an action plan for retrieving the object, that is, a means-ends problem. In order to disambiguate the task, later research has simply presented objects that moves out of sight behind an occluder and observed how the child reacts to those events. This can be done either by measuring their ability to predict where and when the object will reappear or by measuring how their looking times change when some aspect of the events are changed.

Most of this work has been focused on how much infants look at occlusion events in which the spatiotemporal continuity has been violated in some way (for related reviews using this methodology see Spelke, 1994; Mareschal, 2000; Baillargeon, 2004). This has been done by making the object reappear at an unexpected location, not reappear at all, reappear at an unexpected time, or by changing the identity of the object during occlusion. Infants looking durations at these various events are coded online (or later from videotapes) by trained observers. The amount of looking is analyzed, whether it declines when the event is presented several times or whether looking is increased when something happens that is not predictable from the previous events. If the infants look longer at those stimuli, it is concluded that the discrepancy has violated the infants' expectancy. For instance, Baillargeon and associates (Baillargeon et al., 1990; Baillargeon and deVos, 1991; Aguiar and Baillargeon, 1999) habituated infants to a tall and a short rabbit moving behind a solid screen. This screen was then replaced by one with a gap in the top. The tall rabbit should have appeared in the gap but did not. Infants from 2.5 month of age looked longer at the tall rabbit event suggesting that they had expected the tall rabbit to appear in the gap.

These studies indicate that the infants are somehow aware of the motion of a temporary occluded moving object but not exactly how it moves or when it will reappear. For instance it is not clear whether the infants expected the tall rabbit to appear at a specific time or not. The infants might have looked longer because they perceived the identity of the object to be changed. Another problem with this paradigm is that it does not address questions related to the micro organization of looking; only the duration is recorded. In many experiments only one data point is collected per subject. In addition, because this method does not record how infants' goal directed responses relate to occurring events, these studies are unable to inform us of the strengths of infants' knowledge; if these representations are strong enough to guide action.

Measuring infants' actions as they interact with the environment represent a different approach to understanding infants' early perceptual-cognitive development. In this paradigm infants are required to organize their actions towards moving objects that become temporarily occluded. Infant's behavioural responses are recorded and related to the spatial-temporal dynamics of the moving object. With this technique we are able to provide a detailed description of how infant's actions relate to events as they occur. This gives us the opportunity to look at how infants' representations and how their expectations of when and where an occluded object will reappear change over time.

This chapter will attempt to review those studies that have looked at how infants come to organize their own actions towards objects that are temporarily occluded. We will both examine when infants come to represent occluded objects and 
attempt to define those variables that limit (or enhance) infants object representations.

\section{Methodological questions}

Several different behaviours have been used as indicators for infants' ability to represent the spatiotemporal continuity of occluded objects and predict their reappearance. Eye movements are of primary interest but are tricky to measure. They can be coded by human observers from video recordings but this method is very time consuming and crude. More direct, precise, and reliable measurements of where gaze is directed at each point in time are needed. It is possible to measure eye movements with electrooculogram (EOG) which gives very high resolution in time $(>200 \mathrm{~Hz})$, but as infants rarely move just the eyes, the movements of the head need to be measured as well in order to know where gaze is directed. A new generation of eye trackers measure the reflection of infrared light sources on the cornea relative to the centre of the pupil (usually $50 \mathrm{~Hz}$ ). For some of these eye trackers, no equipment is applied to the subject who just sits in front of the apparatus. With appropriate calibration, the measurement of cornea reflection provides precise estimates of where gaze is directed in the visual field.

Using gaze tracking as an indicator of predictive behaviour when the tracked object is occluded, relies on the following considerations. While the object is visible, infants from 2 to 3 months of age tracks it at least partially with smooth pursuit (von Hofsten and Rosander, 1997). When the object disappears behind an occluder the eyes are no longer able to sustain its smooth movements (Leigh and Zee, 1999). Then the observer shifts gaze example of such behaviour can be observed in Fig. 1. The smooth tracking is visible prior to and following the occlusion in Fig. 1B. During the actual occlusion this infant made a saccade from the disappearance edge to the reappearance edge. The timing of this saccade (when the saccade was initiated or when it terminates at the reappearance location) provides information of when the infant expected the object to reappear (for the development of saccade latencies see Gredebäck et al., 2006). The location where the saccade terminates provides information of where the object is expected to reappear. Both of these measures are frequently reported in the following text.

The measurements of arm movements is needed for drawing conclusions about infants ability to direct manual actions towards an occluded moving object. In some studies, video has been used but it is also possible to use more automatic motion capture devices where positions are defined by reflecting markers or light emitting diodes. If the infant reaches for the area where the occluded object will appear before the object emerges from across the occluder in one or more saccades. An

1

B

Fig. 1. (A) An object moving with constant velocity on a circular trajectory that is partly occluded (dark grey areas). (B) Enlargement of a single occlusion passage. The circle represent when the saccade is initiated and the square represents the termination of the saccade. Only horizontal eye movements are displayed. 
1 behind the occluder, then infants are said to be predictive. That is, the infant has then demon-

strated an ability to represent the spatiotemporal properties of the occluded object and the ability to predict how it is going to move in the future. The same logic can be applied to infants' head movements. Moving ones head to fixate the reappearance location ahead of time ensures that the infant fixate the object as it emerges, thereby allowing vision to guide a future reach to the attended object.

\section{At what age do infants start to represent occluded objects?}

A series of early reports were performed by Nelson (1971, 1974) and Meichler and Gratch (1980). In these studies 5- and 9-month-old infants were presented with a toy train that moved around on a track and, at one point, past through a tunnel. Infants watched these events and the experimenter recorded the infants' eye movements with a standard video camera. In summary, the videos of the infants' looking at this event gave no indication that 5-month-old infants anticipated the reappearance of the train from the tunnel. Nine-month olds consistently moved their gaze to the reappearance location at the other end of the tunnel and anticipated the emergence of the train there.

In recent years the technology available to measure infant's eye movements have advanced greatly. Numerous studies have taken advantage of the high temporal and spatial resolution provided by state of the art eye tracking technology. One such early eye tracking study was performed by van der Meer et al. (1994). They investigated 4-12 month-old infants' abilities to predicatively track and reach for an occluded toy which moved on a horizontal plane while measuring the infants' eye movements. Infants first started to reach for the toy at 5 months of age. At this age, infants' reaches were reactively launched at the sight of the reappearing toy. However, at the same age, they moved gaze to the reappearance point ahead of time. Not until infants were 8-month-old did they plan the reaching for the object while it was still occluded. This indicates that anticipatory tracking emerges prior to anticipatory reaching; the former exists from at least 5 months of age.

Recently, Johnson et al. (2003) presented 4month-old infants with objects that become occluded at the centre of the trajectory. These stimuli were presented on a computer monitor and horizontal and vertical eye movements of one eye was recorded using an ASL 504 eye tracker (accuracy 0.5 visual degrees, sampling rate $50 \mathrm{~Hz}$ ). Fourmonth-old infants who had previously been presented with fully visible trajectories (without the occluder) were more likely to predict the reappearance of the object than infants who had not been presented with such learning trials. At 6 months of age infants did not demonstrate the same benefits from seeing non-occluded trials. According to the authors these results demonstrate that 4-month-old infants do not possess robust object representations but that 6-month olds do.

In an attempt to trace the development of predictive looking in the occlusion situation, Rosander and von Hofsten (2004) measured head and eye movements of 7-, 9-, 12-, 17-, and 21-week-old infants as they tracked a real object (a happy face) that oscillated on a horizontal trajectory in front of them. Four different conditions were included in this study. The velocity of the object was either constant or sinusoidally modulated. In the former case the object always moved with the same speed and turned abruptly at the endpoints and in the latter case the object accelerated as it moved towards the centre of the trajectory and decelerated before each turn in a smooth fashion. In addition, the object became occluded for $0.3 \mathrm{~s}$ at the centre of its trajectory or for $0.6 \mathrm{~s}$ at one of the trajectory end points. Trial duration was $20 \mathrm{~s}$ which included five cycles of motion. If the occluder covered the centre of the screen each trial included 10 occlusion events and if the occluder covered the end point each trial included 5 occlusion events. In the latter case, the object reappeared on the same side as where it disappeared.

The level of performance in the central occluder condition improved rapidly over age. The youngest infants were purely reactive. It appeared as if the occluder edge itself became the focus of attention after object disappearance. It was found that the gaze of 7- and 9-week-old infants remained at 
the occluder edge almost $1 \mathrm{~s}$ after the object had reappeared on the other side of the occluder. Thus, in many cases the object had already reversed direction of motion and was approaching the occluder again before the infants re-focused their gaze on the object. The relative inability to quickly regain tracking had more or less disappeared for the 12-week olds. At that age, infants moved gaze to the reappearance point as soon as the object became visible (that is after $\sim 0.5 \mathrm{~s}$ ). Furthermore, the 12-week olds showed signs of being able to represent the moving object after having seen several occlusions. The mean gaze lag at reappearance for the last cycle of the trial with the triangular motion was predictive (see Fig. 2). The fact that also the younger infants became more aware of the reappearing object with experience over a trial suggests that they acquired some kind of representation of the occluded object.

The infants had an increasing tendency with age to extrapolate the occluded motion to the other side of the occluder when it was placed over one of the end points of the trajectory. For the 21-week olds, this tendency was dependent on the motion function used for the oscillation. When the object moved with constant velocity (triangular motion), the subjects made more false gaze shifts to the other side of the occluder. In this condition, there is no way to determine from a single occlusion event whether the object is going to continue or reverse its motion behind the occluder.

To summarize, these studies are all groundbreaking in their own right. The early studies by Nelson $(1971,1974)$ were the first to measure gaze tracking during occlusion and to demonstrate the importance of learning in occlusion events. The first study to look at eye-hand interaction during occlusion in infancy was provided by van der Meer et al. (1994). At the same time Johnson et al. (2003) and Rosander and von Hofsten (2004) pinpoint the immense importance of previous experiences. Johnson et al. focused on prior experiences with non-occluded objects whereas Rosander and von Hofsten provided a unique illustration that development does not consist of multiple hierarchal knowledge categories. Instead development of object representations is a continuous process that begins as early as 7 weeks-of-age and continuous far beyond 5 months-of-age.

As such, all fail-proof statements about when infants come to represent and predict occluded objects must be regarded with scepticism. Instead the effects of each study that report on the emergence of object representations must be seen in the context of prior experiences (both with fully visible and occluded trials). It should be noted, however, is that each of these reports demonstrated a similar onset of object representations at 4 months of age. This is valid even for the study by Johnson et al. (2003); they reported an increase in predictive tracking with prior experience at 4 months of age. This learning appears to be a fundamental component of object representations and should (quite opposite to the authors interpretation) be interpreted in support of the notion that 4-month olds have developed such an ability. To date no study has reported on consistent predictive responses at an earlier age.

\section{Mapping out the psychometric space}

\section{Trajectory parameters}

Clearly the learning effects described above are not the only component that defines if infants will display mature object representations and have the ability to predict the reappearance location of occluded objects. The ability to represent an occluded object is also dependent on the velocity and amplitude of the moving object and on the duration of the current occlusion event (to name a few contributing factors). The fact that different parameters of the ongoing object motion (independent of previous experiences) is important for infants abilities to predict the reappearance location of occluded objects is nicely illustrated by two studies performed by Gredebäck and von Hofsten (Gredebäck et al., 2002; Gredebäck and von Hofsten, 2004).

In these studies 6-12 month old infants and adults were presented with an object that moved on a circular trajectory and became occluded once every lap. The study by Gredebäck and von Hofsten (2004), for example, presented such circular 


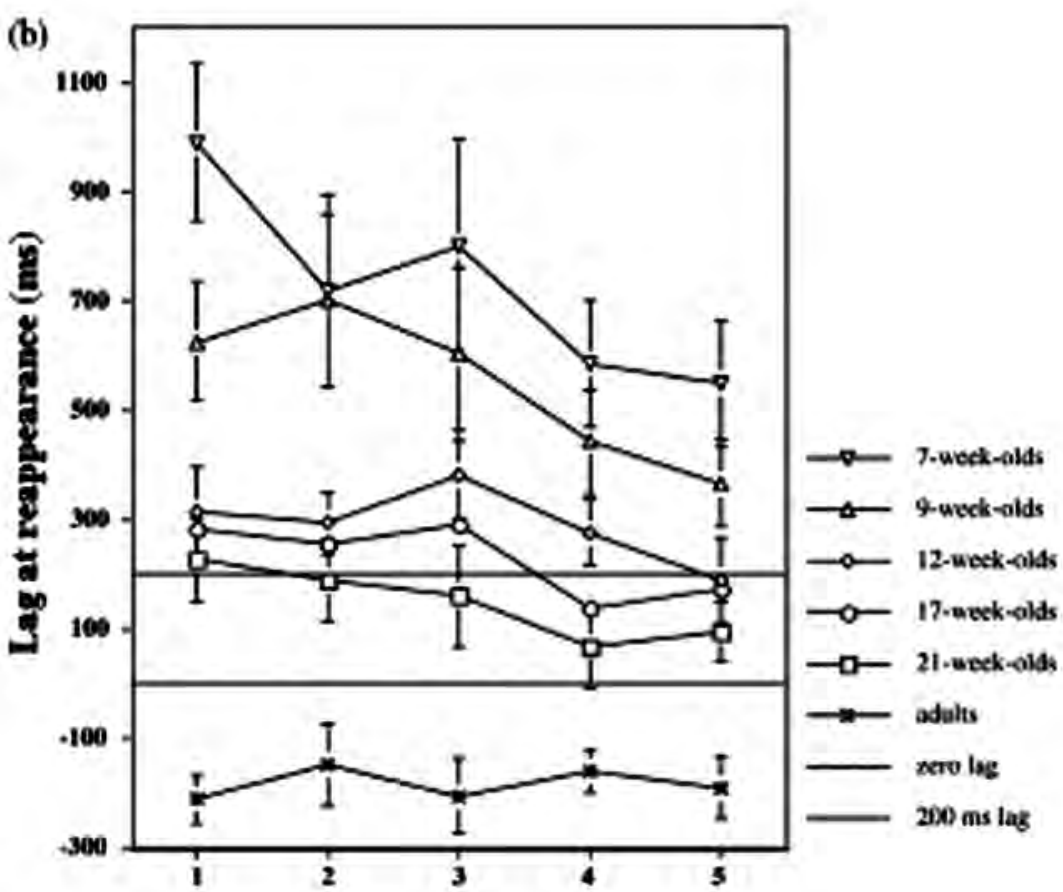

Cycle
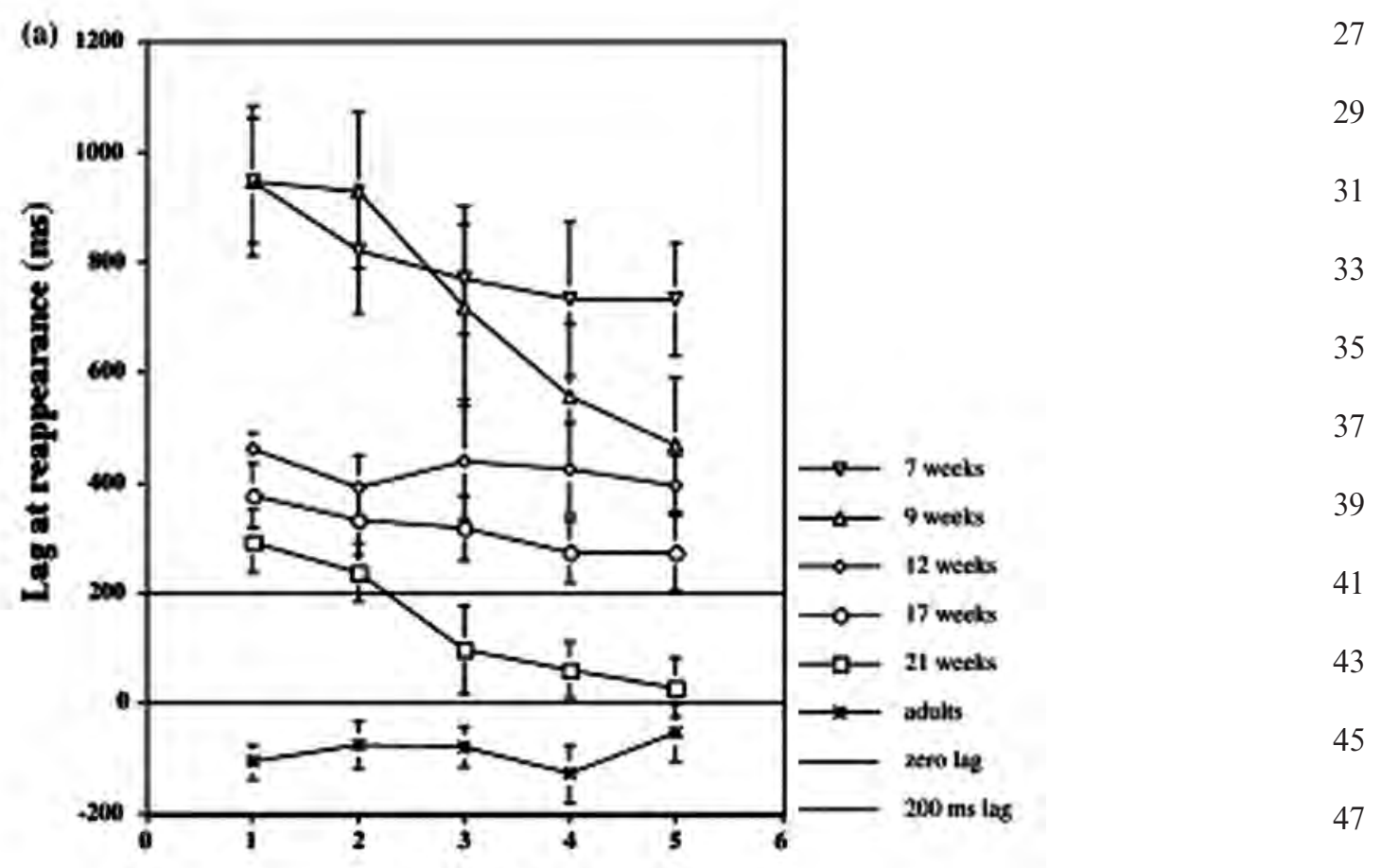

\section{Cycle}


1 trajectories to a group of infants that was followed longitudinally from 6 to 12 months of age. In this study the size of the occluder always remained the same $(20 \%)$ but velocities of the moving object varied $\left(2.5-20^{\circ} / \mathrm{s}\right)$; resulting in four occlusion durations ranging from 500 to $4000 \mathrm{~ms}$. Both studies randomized the presentation order of the different occlusion event and used an ASL 504 eye tracker to measure gaze direction.

The combined experience from these studies is that infants often failed to predict the reappearance of the target (for proportion of successful predictions see Fig. 3), even at 12 months of age (adults performed perfectly). Surely, the between trial randomization lowered the overall performance level and the circular trajectory probably made it more difficult to represent the trajectory of the target. However, the finding illustrated in Fig. 3 is that infants' performance at each age was highly influenced by the velocity (and/or occlusion duration) of the target. The 12-month-old group, for example, ranged in performance from $<20 \%$ to $>80 \%$ predictions dependent on the stimuli used. Unfortunately, these studies cannot disentangle if the occlusion duration or the velocity of the target is the driving factor behind this change (since they co-vary).

However, a recent study by von Hofsten et al. (in press) presented 4-month-old infants with a series of sinusoidal horizontal trajectories (randomized between trials). The design systematically varied occluder width, amplitude of the motion, and velocity of the moving object independently of each other. This was done in order to understand which variables contributed to infants' ability to represent and predict the objects reappearance during occlusion. The results demonstrated that infant's performance could not be explained by occluder edge salience, occluder duration on previous trials, or simply the passage of time. They rather geared their proactive gaze shifts over the occluder to a combination of occluder width, oscillation frequency, and motion amplitude that resulted in a rather close fit between the latency of the proactive gaze shifts and occlusion duration. Instead of having explicit knowledge of the relationship between these variables, infants could simply maintain a representation of the object motion and its velocity while the object is occluded. The results of von Hofsten et al. (in press) strongly supported this hypothesis. This can be seen in Fig. 4. It is as if the infants tracked an imagined object in their 'minds eye'. If object motion is represented in this way during occlusion, the effects of occluder width, oscillation frequency, as well as motion amplitude can all be explained.

In summary, numerous variables associated with the ongoing occlusion event determine how well an infant will be able to predict the objects reappearance. Even 12-month-old infants often fail to predict the reappearing object if the velocity is high and the trajectory circular. The final study described above (von Hofsten et al, in press) made it abundantly clear that object representations are dependent on numerous simultaneous factors associated with the ongoing occlusion event. These findings clearly demonstrate the importance of mapping out the multidimensional psychometric space that governs object representation and the ability to perform an accurate prediction.

\section{What stimulus information defines occlusion?}

In the study by Gredebäck and von Hofsten (2004), we argued that infant's difficulties with high velocities could not result from an inability to track fast moving objects. Quite the contrary, we found that infants track (gaze and smooth pursuit) similar fast non-occluded motion with higher accuracy (timing and gain) than slower motion (Gredebäck et al., 2005; Grönqvist et al., 2006). Instead we argued that these difficulties can be related to the duration (clarity) of the gradual

Fig. 2. The average time differences and SE between object and gaze reappearance at each cycle of the centrally occluded trials. Separate graphs are shown for the sinusoidal (a) and the triangular motion (b). Each data point is the average of one occluder passage in each direction for all subjects in a specific age group. The upper line corresponds to the minimum time required for adults to program a saccade to an unexpected event $(200 \mathrm{~ms})$. Adapted with permission from Rosander and von Hofsten (2004). 
6

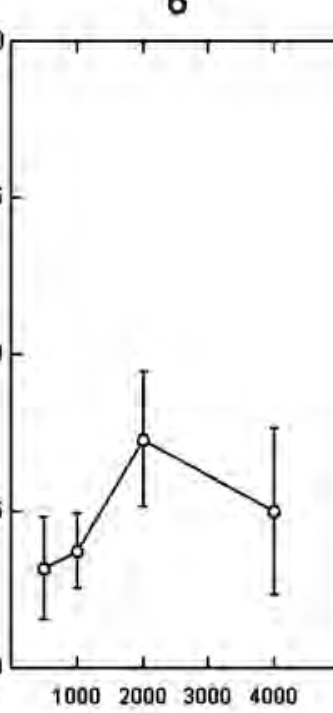

8

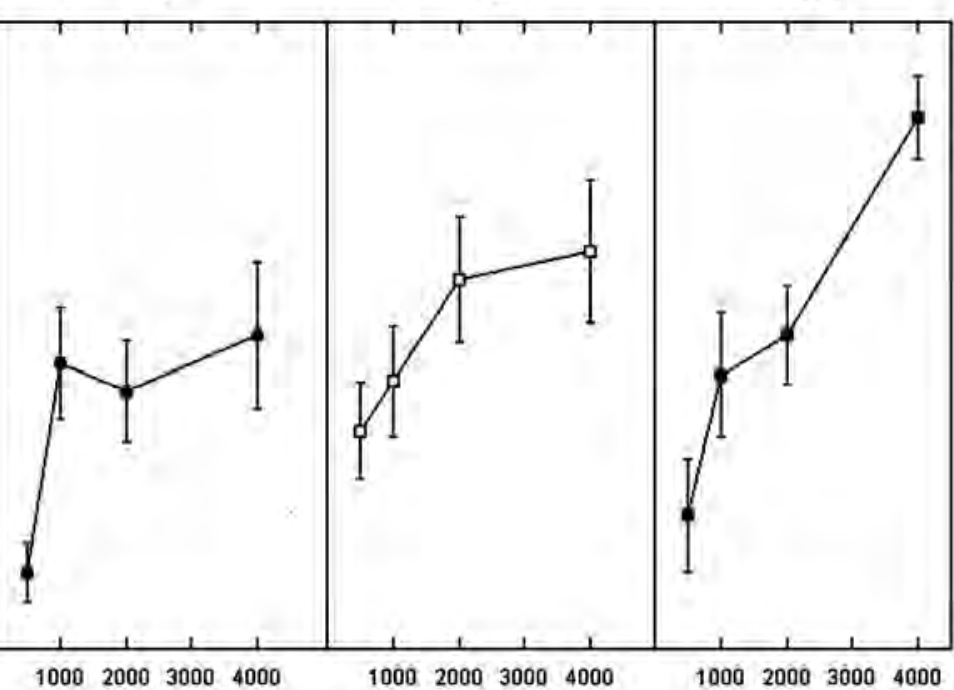

occlusion duration (ms)
1 during the occlusion event at the same time as one maintains both occlusion durations and identical pre- and post-occlusion trajectories. Such studies were performed by von Hofsten et al. (2000) and Jonsson and von Hofsten (2003). Jonsson and von Hofsten (2003) measured 6-month-old infant's head tracking and reaching during occlusion and blackout. During these events a target moved on a straight horizontal path in front of the infants. Either the object was fully visible during the entire

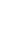

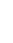

列

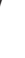

9

1

3

5

7

19

21

23

25

27

29

31

33

35

37

39




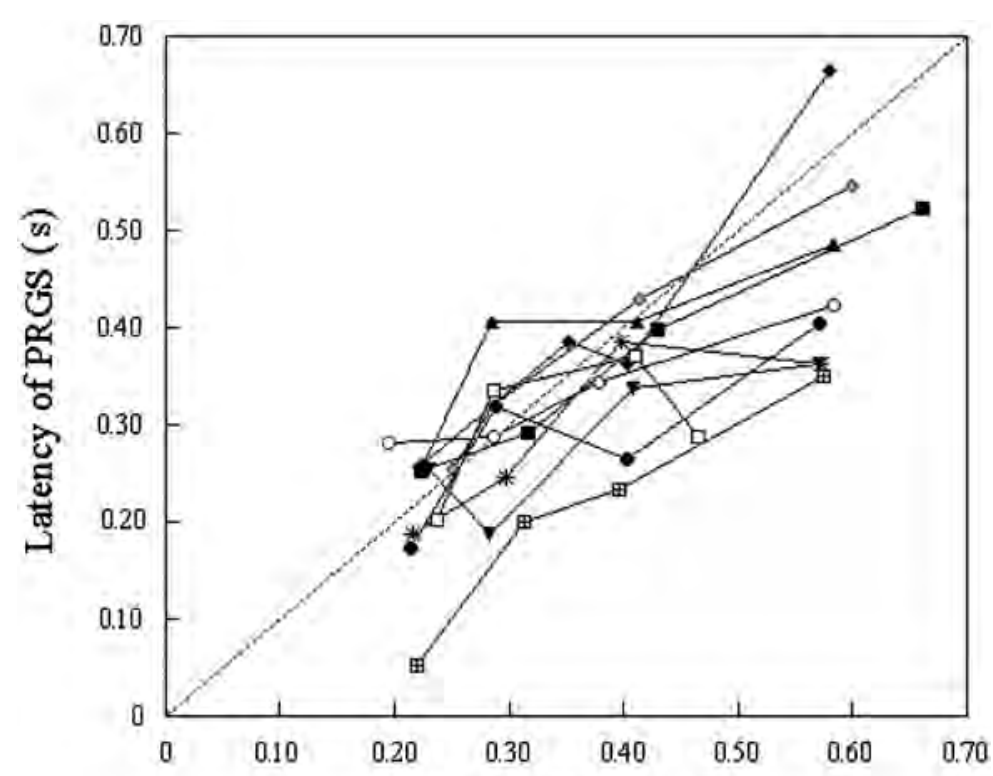

Occluder duration (s)

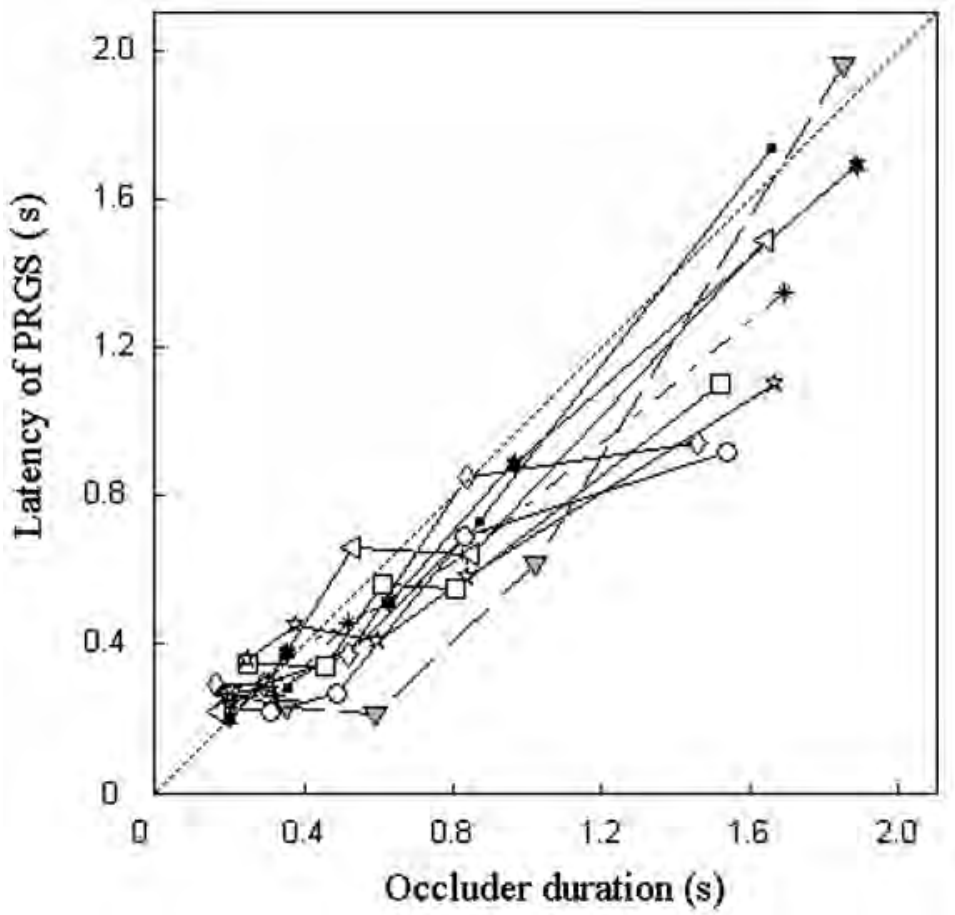

Fig. 4. (a) The relationship between occlusion duration and proactive saccades for individual subjects in Experiment 1 that included occlusion durations of from 0.22 to $0.61 \mathrm{~s}$. (b) The relationship between occlusion duration and proactive saccades for individual subjects in Experiment 2 that included occlusion durations from 0.2 to $1.66 \mathrm{~s}$. The dashed line in both figures shows the hypothetical relationship with saccade latency equal to occlusion duration. Adapted with permission from von Hofsten et al. (in press). 
Fig. 6. Colour histograms that include all data points recorded (combined over the two conditions) at each age. Data from each age group is divided in to three pictures dependent on the location of the ball. In between each stimulus infants were presented with an attention-grabbing movie at the centre of the screen. Initial fixations at this location before infants moved their gaze to the ball and started tracking is visible at the centre of the screen when the target is visible to the left.

trajectory or it became invisible during a period just prior to the optimal reaching space. Three different occlusion durations were used in combi-

Fig. 5. (A) The deletion condition, (B) the shrinking condition, (C, D) the areas where the ball successively disappear and reappear. head tracking was more inhibited by blackout than by a visible occluder but the opposite effect was observed during reaching. No consistent effects of occlusion duration were observed during blackout. 
During occlusions, however, the head led at first target reappearance (predictions) and the size of the mean lead increased with prolonged duration of non-visibility.

In summary, these studies add another factor that limits object representations; namely the stimulus information that defines occlusion. The study by Gredebäck et al. (in prep.) demonstrates that providing a clear deletion event allows infants to classify the stimulus as an occlusion event, and this will in turn, strengthens infant's representations and promote predictions. The study by Jonsson and von Hofsten (2003) demonstrated that the manner in which an object is obstructed from view (occlusion or blackout) also influence the way in which infants are able to deal with the object in its visual absence. Head tracking is more disrupted by competitive visual stimuli (the occluder) and is less disrupted by blackout. Clearly infants' actions on objects that are temporarily out of view are not only influenced by the structure of the stimuli but also by the manner in which it disappears from view.

\section{How specific are object representations?}

Several studies indicate that infants' ability to represent occluded objects in the context of reaching is much inferior to their ability to represent them in the context of looking (Spelke and von Hofsten, 2001; Jonsson and von Hofsten, 2003; Hespos et al., submitted). Spelke and von Hofsten (2001) and Jonsson and von Hofsten (2003) found that predictive reaching for occluded objects were almost totally absent in 6-month-old infants. At the same time they did not seem to have problems with tracking them with their head (von Hofsten et al., 2000; Jonsson and von Hofsten, 2003).

Hespos et al. (submitted) recorded the predictive reaching of 6- and 9-month-old infants who viewed an object that moved in a straight line and, on some trials, was briefly occluded before it entered the reaching space. While there was an increase in the overall number of reaches with increasing age, there were significantly fewer predictive reaches during the occlusion trials than during the visible trials and this pattern showed no age- related change. In a second experiment, Hespos et al. developed a reaching task for adults modelled on the tasks used to assess predictive reaching in infants. Like infants, the adults were most accurate when the target was continuously visible and significantly less accurate when the target was briefly occluded. These findings suggest that the nature and limits to object representations are similar for infants and adults.

Following Shinskey and Munakata (2003) and Scholl (2001), Spelke and von Hofsten (2001) suggested that young infants represent both visible and hidden objects, and their object representations depend on the same mechanisms as those used to represent and attentively track objects in adults (Scholl, 2001). More specifically, the object representations of infants and adults have three properties. First, these representations are more precise, at all ages, when objects are visible than when they are hidden. Second, representations of different objects are competitive; the more objects one attends to, the less precise will be one's representation of each object. Third, precise representations are required for reaching: to reach for an object, one must know where it is, how big it is, what shape it is, and how it is moving. In contrast, less precise representations suffice to determine that a hidden object exists behind an occluder in a scene that one observes but does not manipulate.

Spelke and von Hofsten (2001) proposed that object representations change over human development in just one respect: They become increasingly precise. Just as infants' sensory and perceptual capacities become more accurate with age (e.g. Kellman and Arterberry, 1998), so does their capacity to represent objects. While infants may reliably predict the reappearance of an occluded moving object moving on a linear path from 4 months of age, the ability to predict where and when the moving object will reappear from behind an occluder is problematic to children beyond their first birthday (Gredebäck and von Hofsten, 2004). Both visible and occluded objects are therefore represented with increasing precision as infants grow.

These properties suffice to account for all the reviewed findings. Object representations are more precise in the dark than in the presence of a visible 
1 occluder, because the occluder competes with the hidden object for attention, decreasing the preci-

sion of both object representations. When a young infant participates in a preferential looking experiment involving an occluded object, moreover, she can draw on her imprecise representation of the object to determine that it exists behind the occluder, and in addition identify gross properties of the object such as its approximate location (e.g. Baillargeon and Graber, 1988) and the orientation of its principal axis (Hespos and Rochat, 1997). Nevertheless, a young infant is likely to fail to represent the exact shape, size, or location of an occluded object, because his or her representation is less precise than that of an older child. When a young infant is presented with an occluded object in a reaching experiment, this same imprecise representation is not sufficient to guide object-directed reaching. The differing precision required by many preferential looking experiments vs. many reaching experiments therefore can account for their different outcomes.

\section{Can infants learn new rules of object motion?}

We know that infants can extrapolate linear horizontal trajectories from at least 4 months of age (see the discussion on the emergence of object representations above) and that infant's actual performance on a given trial is dependent on the structure of the perceived events, their previous experiences, and the manner in which the object disappears. We also know that infants from at least 6 months of age can extrapolate circular trajectories (Gredebäck et al., 2002; Gredebäck and von Hofsten, 2004). In these studies (reviewed above) both infants' and adults' predictive saccades terminated along the curvature of the circular trajectory (at the reappearance edge of the occluder).

So, we conclude from these studies that infants extrapolate a number of different naturally occurring trajectories. However, what is still unknown from the above-mentioned studies is whether infants can construct new rules of novel trajectories or if infants are solely governed by pre-existing knowledge of how objects naturally move. This question, whether the ontogenetic origin of infants object representations emerge from innate knowledge structures (nativism) or if this knowledge emerge in an interaction with the environment (constructivism) have recently been the focus of much research.

The first two studies to address this issue (while relating the infants' predictions to the actual reappearance location of the object) were performed by von Hofsten and Spelke (von Hofsten et al., 2000; Spelke and von Hofsten, 2001). In these studies the authors measure 6-month-old infants' predictive reaching and head tracking during an occlusion task.

In both studies infants were seated in front of a vertical surface on which a toy moved on linear paths. Half of all trials started with the target moving from the upper edges of the screen, moving downwards on a diagonal path (linear trials). During other trials the toy started moving in the same manner but changed direction at the centre of the screen; continuing downwards but reversing the horizontal direction (non-linear trials). At the intersection between these trajectories (the centre of the screen) the toy moved behind an occluder (see Fig. 7). This event prevented the infants from perceiving whether the toy moved on a straight or

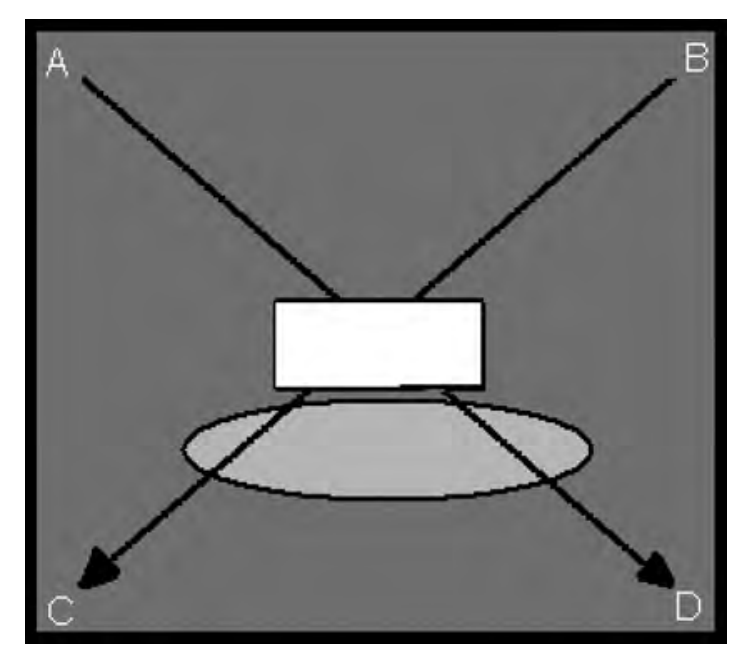

Fig. 7. Arrows and letters indicate the four trajectories used $(\mathrm{A} \rightarrow \mathrm{D}, \mathrm{B} \rightarrow \mathrm{C}, \mathrm{A} \rightarrow \mathrm{C}, \mathrm{B} \rightarrow \mathrm{D})$. The white square indicate the approximate location of the occluder while the light grew ellipse represent the optimal reaching space of infants. 
turning trajectory. To predict the reappearance of the object, the infants had to turn their head either to the lower right or left side of the occluder (occlusion durations were 400 and $900 \mathrm{~ms}$ ). Spelke and von Hofsten (2001) contrasted these occlusion events with fully visible trials.

During the first occlusion event infants did not anticipate the reappearance of the toy. However, with experience infants rapidly predicted the reappearance on linear trials (after three trials). Even non-linear trials were anticipated, but learning was slower. These studies demonstrate that 6-monthold infants' are better equipped to learn about linear trajectories than they are to learn about non-linear trajectories. This finding was interpreted in support of the nativist view; suggesting that infants have a pre-existing notion that objects naturally move on linear trajectories (e.g. inertia) and that infants use this knowledge to extrapolate the pre-occlusion trajectory.

In retrospect, these papers (von Hofsten et al., 2000; Spelke and von Hofsten, 2001) demonstrate something different altogether. The studies suggest that infants have multiple strategies available to solve an occlusion task. Infants can extrapolate the pre-occlusion trajectory but they also have the ability to learn how to predict novel (non-linear) trajectories. As such, these studies do not inform us about the ontology of infants object representations but illustrate the diversity of recourses available to an infant when faced with an occlusion event.

To better understand the nature of these two forms of prediction, Kochukhova and Gredebäck (in press) presented infants with movies in which a ball rolled back and forth between two endpoints. The middle of the trajectory was covered by a round occluder. Eye movements were measured with a Tobii eye tracker. Experiment 1 compared infants' ability to extrapolate the current pre-occlusion trajectory with their ability to base predictions on recent experiences of novel object motions. In the first (linear) condition infants were presented with multiple linear trajectories. These could be extrapolated but infants were unable to rely on memories of previous events to solve the occlusion task (since each session included multiple trajectories with different

directions of motion). In the second (non-linear) condition infants were presented with multiple identical trajectories that turned $90^{\circ}$ behind the occluder. These trajectories could not be extrapolated but infants were able to rely on previous experience to predict where the target would reappear.

In the linear condition infants performed at asymptote $(\sim 2 / 3$ accurate predictions) from the first occlusion passage and performance did not change over the session. In the non-linear condition all infants initially failed to make accurate prediction. Performance, however, reached an asymptote after two occlusion passages. This initial experiment demonstrates that infants have an initial assumption that objects will continue along the linear extension of the pre-occlusion trajectory. But the results also demonstrate that infants can change their predictions if another source of information is more reliable.

In a second experiment the learning effect observed in response to the non-linear trajectories were replicated and extended. Here infants were presented with the same set of non-linear trajectories on three different occasions; a first session as soon as they arrive in the lab, a second session after a 15 min break, and a third session $24 \mathrm{~h}$ later. The results can be observed in Fig. 8 .

First of all, infants quickly learned to predict the correct reappearance location of the ball. However, after a $15 \mathrm{~min}$ break infants had completely forgotten where the ball reappeared. Infants required a second session to consolidate their experience and form a stable memory of where the ball would reappear. After this second session infants were able to maintain a representation of the trajectory for at least $24 \mathrm{~h}$.

This final study demonstrates that infants' initial assumptions are consistent with a linear extension of the pre-occlusion trajectory. But, more importantly, the study demonstrates that infant can acquire new knowledge after only a few presentations and have the ability to maintain this information over time. We suggest that these different approaches to solving an occlusion task (extrapolations and memories of previous events) are not governed by separate mechanisms. Instead we interpret these findings in support of the 


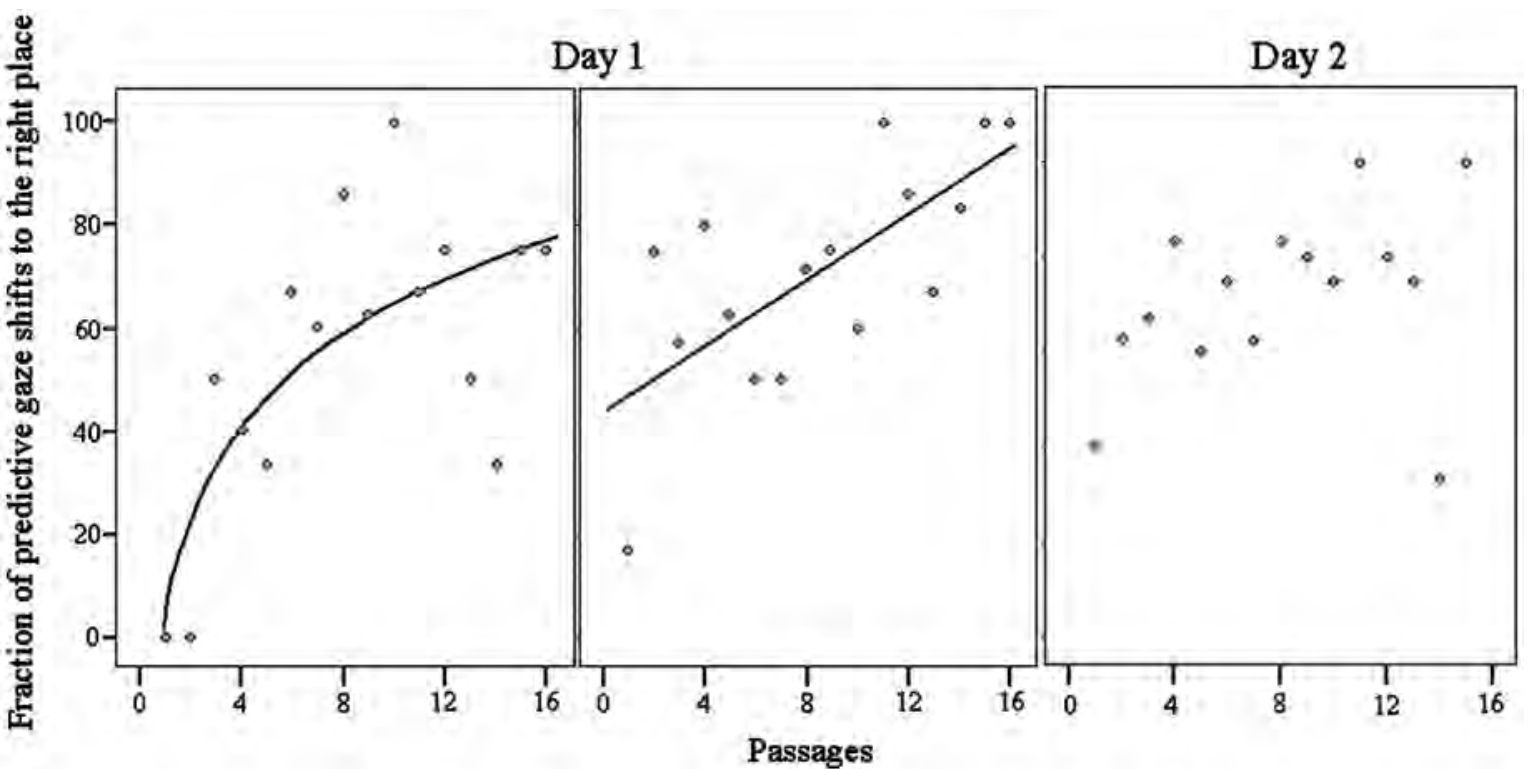

Fig. 8. Percentage of predictive occlusion passages that appear in the correct reappearance location in each of the three sessions of Experiment 2 of Kochukhova and Gredebäck (in press). Each dot represents the average percent accurate predictions on that occlusion passage. Lines depict the regression line with most explained variance; no significant changes were observed during the second day.

constructivist view, suggesting that both steam

from the infants' own experience with the environment. Infants learn to predict non-linear trajectories in the lab but have most likely had enough experience with linear (and curvilinear) trajectories in the real world to help them formulate a valid hypothesis about how objects naturally move. From this perspective the current results appear almost trivial; infants are initially more proficient with extrapolation since this is the only trajectory (of the two presented) that infants have had any real experience with (prior to the study). After a number of presentations of non-linear trajectories infants learn to predict these with equal proficiency.

\section{What does prediction really mean?}

One noteworthy aspect of measuring anticipatory gaze shifts during occlusions is that predictions occur on only about half of all presented trials in infancy. Despite this, we claim that infants from at least 4 months of age can represent occluded objects. In Rosander and von Hofsten (2004) and in von Hofsten et al. (in press) the 4-month-old infants moved gaze over the occluder ahead of time in $47 \%$ of the trials and in Johnson et al. (2003) in $29-46 \%$ depending on condition and age. Similar levels of anticipatory gaze shifts have been observed at 6- (Kochukhova and Gredebäck, in press) and 12-month-old infants (Gredebäck and von Hofsten, 2004). If infants track the spatiotemporal contiguity of the occluded object why do they not make accurate predictions on every trial?

First of all, there is no way to ask infants to pay attention to a specific aspect of the visual scene. As an obvious effect thereof, infants will, on occasion, disrupt tracking and look at some non-task related aspect of their visual scene. If infants shift their attention away from the moving object during occlusion then some of these trials will be undistinguishable from a reactive trial (in which the infant only fixated the moving object after it has reappeared from behind the occluder). It is therefore likely that the above-mentioned studies underestimate infants' performance to some degree.

In addition to voluntary changes in attention, infants' ability to actually represent the occluded object is dependent on the relative salience of each 
aspects of the visual field. As mentioned above, the different elements of the visual field (visible and hidden) compete with each other for available recourses. When a moving object is occluded the relative saliency of visible stimuli (like the occluder) increases. According to this logic, infants might have a general ability to represent non-visible objects but the actual performance on a given trial is easily disrupted.

We have described a number of studies that demonstrate the diversity of infants' performance and the highly variable results obtained through small changes in the psychometric space that make up the visual scene and the occlusion event. Each of these components (e.g. the occluder width, the way the object disappears, and the amplitude of the trajectory) independently influence the relative representational strength of the occluded object and its surroundings. Each helps build up and/or degrade object representations in a non-linear fashion.

\section{Myths about eye tracking and occlusion}

This chapter has reviewed a number of studies that measured infants' abilities to predict the reappearance of occluded objects. All of these studies rely on the assumption that predictions are synonymous with (or at least related to) infants' abilities to represent the occluded object and/or its spatiotemporal dynamics. There are, however, a few rival interpretations of these findings. Interpretations that questions the link between prediction and representations, especially when infant's eye movements are used as a dependent measure. The following paragraphs will introduce these alternative interpretations and address why they are unable to account for the obtained results.

\section{Could predictive gaze shifts be the result of random looking?}

Is it possible that infants stop tracking at the occluder edge when the object disappears, wait there for a while and then shift gaze anyway in a random fashion. Some of those spontaneous gaze shifts might arrive to the reappearance side of the

occluder before the object reappears there. Such random tracking would provide a number of false predictions. Three of the above-mentioned studies clearly demonstrate that this is not the case.

The study by Gredebäck and von Hofsten (2004) presented infants with four different occlusion durations. In this study infants scaled their proactive gaze shifts over the occluder to the actual occlusion duration. More gaze shifts were made after $400 \mathrm{~ms}$ in response to a $500 \mathrm{~ms}$ occlusion event than in response to a 1000 ms occlusion event, and a similar relationship existed for each of the four-occlusion durations. If gaze moved at random, then the same number of gaze shift would end up on the reappearance side of the occluder independent of the actual occlusion duration. In a similar vain, von Hofsten et al. (in press) demonstrated that the proportion of gaze shifts to the reappearance edge ahead of time showed no relationship with occlusion duration in either of the two experiments. Again, the proportion of gaze shifts ending up at the reappearance side of the occluder would increase with prolonged occlusion durations if infants gaze shifts were launched and directed at random.

A third example comes from the study by Kochukhova and Gredebäck (in press). In this study, the number of gaze shifts during occlusion to each side of the occluder was compared. Infants were only judged to have the ability to predict the actual reappearance location if they made more gaze shifts to this location compared to the alternative reappearance locations along the occluder edge. Their ability to move to the correct location was dependent on the trajectory being presented and on their previous experience with similar events. If infants had moved their gaze at random, then each side of the occluder would be fixated to an equal degree and none of these effects would be significant.

\section{Could predictive gaze shifts be the result of occluder salience?}

This alternative account suggests that the salience of the occluder's reappearance edge determine whether infants make predictive saccades across 
the occluder. If this was the case then stimuli with greater visual salience would attract attention to a higher degree and result in earlier gaze shifts. As contrast sensitivity decreases with increasing eccentricity in the visual field, it is possible that gaze shifts in the presence of a wide occluder will have a longer latency, not because the subject expects the object to reappear later, but because the visual salience of the exiting occluder edge is then lower. One argument against the visual salience hypothesis comes from the reactive saccades in the study by von Hofsten et al (in press). Reactive saccades are by definition elicited by the detection of the reappearing object in the periphery of the visual field. In this study von Hofsten et al. found that the effect of occluder width on reactive saccade latency was small $(0.45 \mathrm{~s}$ for the narrow and 0.54 for the wide) in comparison to the difference in the latency of the proactive saccades $(0.33 \mathrm{~s}$ for the narrow and 0.79 for the wide occluder). It is therefore unlikely that it is the visual salience of the exiting occluder edge that determines the difference in saccade latency for the different occluder widths. One can, of course, argue that a non-salient stimulus in the periphery of the visual field like the occluder edge will take longer to detect than a salient one like the reappearing object. However, the latency of proactive saccades for the narrow occluder was shorter than the reaction time to the salient reappearing object in the same condition. Finally and most importantly, visual salience could not be the only determinant of the proactive saccades. The effects of oscillation frequency and motion amplitude were found to be just as important. Motion amplitude and oscillation frequency refer to variables that are not visually present during occlusion and therefore it is inevitable that information from the seen pre-occlusion motion is preserved during occlusion.

\section{Could predictive gaze shifts be the result of conditioning?}

This alternative account of the studies reviewed above suggests that predictive saccades are the result of the simple contingency between disappearance and reappearance locations. The hypothesis is derived from operant conditioning and does not involve any representational abilities. At least three of the above-mentioned studies clearly demonstrate that this is not the case. The strongest evidence against this alternative hypothesis comes from the study by Kochukhova and Gredebäck (in press). In the first experiment of this study infants were presented with a numerous linear trajectories with different disappearing and reappearing position. Each trajectory was randomly selected from a set of linear trajectories leaving no room for conditioning of location. Despite this, infants performed at asymptote from the very first trial. The fact that infants predicted the linear trajectory the first time they saw the stimuli clearly indicates that conditioning cannot account for infants' predictions.

The same conclusion can be drawn from the study by Gredebäck and von Hofsten (2004). In their study infants were presented with four different (randomized) occlusion durations and that made conditioning of occlusion duration near impossible.

A third example comes from von Hofsten et al. (in press). They measured whether the previous occlusion duration had an impact of the latency of infants' saccade across the occluder on the current trial. No such factor emerged in the analysis instead infants performance was guided by parameters of the current occlusion event.

\section{Summary}

The reviewed research demonstrates that infants' actions are directed to the reappearance of occluded objects from a very early age. At around 4 months of age, infants overcome the temporary occlusion of an object they track by shifting gaze ahead of time to the position where it reappears. Before this age, infants have not demonstrated an ability to predict the reappearance of occluded objects but they still benefit from experience; decreasing their reactive saccade latencies over successive passages from the earliest age tested (7 weeks of age). Occlusion is not only problematic to young infants; they appear to challenge even the adult mind. 
We also demonstrate that prediction is not an all or none process that infants either lack or possess. Instead each infant's abilities to predict the reappearance of an occluded object are dependent on numerous simultaneous factors. These include parameters of the current occlusion event (e.g. oc-

clusion duration and the manner in which the object disappears) and previous experiences with similar events (both within the current trial and more long-term experience that predate the experimental session). This illustrate that infant's abilities to predict the motion of an occluded object is determined, in part by their own representational abilities, but also by the dynamics of the current occlusion event, and the relative representational strengths of visible and occluded objects.

We have argued that infants' understanding of how occluded objects move is based on prior experiences with similar events. The functioning of basic biological processes like those related to the perception of object velocity and accretion/deletion at an occluder edge are necessary for allowing the infant to be aware of the object when it is out of sight. We propose that these principles are acquired through an interaction with the environment. Infants will initially extrapolate the trajectories of occluded objects because they have massive experience with linear (and curvilinear) trajectories. But infants also have the ability to rapidly adjust to novel trajectories that violate their initial expectations. All of these findings support a constructivist view of infants' object representations.

\section{Acknowledgements}

This paper was supported by Swedish Research Council (421-2006-1794) and the Norwegian Directorate for Children, Youth, and Family Affairs (174038).

\section{References}

Aguiar, A. and Baillargeon, R. (1999) 2.5 month-old infant's reasoning about when objects should and should not be occluded. Cognit. Psychol., 39: 116-157.
Baillargeon, R. (2004) Infants' reasoning about hidden objects: evidence for event-general and event-specific expectations. Dev. Sci., 7(4): 391-414.

Baillargeon, R., Graber, M., Devos, J. and Black, J. (1990) Why do young infants fail to search for hidden objects? Cognition, 36(3): 255-284.

Baillargeon, R. and deVos, J. (1991) Object permanence in young infants: further evidence. Child Dev., 62(6): 1227-1246.

Gibson, E.J. and Pick, A.D. (2000) An Ecological Approach to Perceptual Learning and Development. University Press, Oxford.

Gredebäck, G. and von Hofsten, C. (2004) Infants' evolving representation of moving objects between 6 and 12 months of age. Infancy, 6(2): 165-184.

Gredebäck, G., von Hofsten, C. and Boudreau, J.P. (2002) Infants' visual tracking of continuous circular motion under conditions of occlusion and non-occlusion. IBAD, 25: 161-182.

Gredebäck, G., von Hofsten, C., Karlsson, J. and Aus, K. (2005) The development of two-dimensional tracking: a longitudinal study of circular pursuit. Exp. Brain Res., 163(2): 204-213.

Gredebäck, G., Örnkloo, H. and von Hofsten, C. (2006) The development of reactive saccade latencies. Exp. Brain Res., 173(1): 159-164.

Hespos, S.J. and Rochat, P. (1997) Dynamic mental representation in infancy. Cognition, 64: 153-188.

von Hofsten, C., Fenq, Q. and Spelke, E.S. (2000) Object representation and predictive action in infancy. Dev. Sci., 3(2): 193-205.

von Hofsten, C., Kochukhova, O. and Rosander, K. (in press). Predictive tracking over occlusions by 4-month-old infants. Dev. Sci.

von Hofsten, C. and Rosander, R. (1997) Development of smooth pursuit tracking in young infants. Vision Res., 37(13): 1799-1810.

Johnson, S.P., Amso, D. and Slemmer, J.A. (2003) Development of object concepts in infancy: evidence for early learning in an eye-tracking paradigm. PNAS, 100(18): 10568-10573.

Jonsson, B. and von Hofsten, C. (2003) Infants ability to track and reach for temporarily occluded objects. Dev. Sci., 6: 88-101.

Kellman, P.J. and Arterberry, M. (1998) The Cradle of Knowledge: Development of Perception in Infancy. MIT Press, Cambridge, MA.

Kochukhova, O. and Gredebäck, G. (in press). Learning about occlusion: initial assumptions and rapid adjustments. Cognition.

Leigh, R.J. and Zee, D.S. (1999) The Neurology of Eye Movements (3rd ed.). Oxford University Press, New York, NY.

Mareschal, D. (2000) Object knowledge in infancy: current controversies and approaches. TICS, 4(11): 408-416.

van der Meer, A.L.H., van der Weel, F.R. and Lee, D.N. (1994) Prospective control in catching by infants. Perception, 23: 287-302. 
Meichler, M. and Gratch, G. (1980) De 5 month-olds show object conception in Piaget's sense? IBAD, 3: 256-281.

Nelson, K.E. (1971) Accommodation of visual tracking in human infants to object movement patterns. J. Exp. Child Psychol., 12: 182-196.

Nelson, K.E. (1974) Infants' short-term progress towards one component of object performance. Merril-Palmer Q., 20: 3-8.

Piaget, J. (1954) The Origin of Intelligence in Children. Routledge, New York.

Rosander, K. and von Hofsten, C. (2004) Infants' emerging ability to represent occluded object motion. Cognition, 91(1): $1-22$.

Scholl, B. (2001) Objects and attention: The state of the art. Cognition, 80: 1-46.

Shinskey, J.L. and Munakata, Y. (2003) Are infants in the dark about hidden objects? Dev. Sci. 6: 273-282.

Spelke, E.S. (1994) Initial knowledge: six questions. Cognition, 50: 431-445.

Spelke, E.S. and von Hofsten, C. (2001) Predictive reaching for occluded objects by 6 month old infants. J. Cognit. Dev., 2: 261-282.
1

3 
AUTHOR QUERY FORM

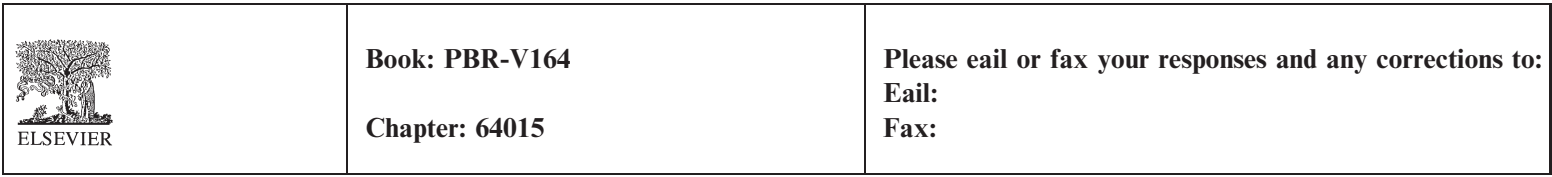

9 Dear Author,

During the preparation of your manuscript for typesetting, some questions may have arisen. These are listed below. Please check your typeset proof carefully and mark any corrections in the margin of the proof or compile them as a separate list*.

Disk use

Sometimes we are unable to process the electronic file of your article and/or artwork. If this is the case, we have proceeded by:

$\square$ Scanning (parts of) your article $\square$ Rekeying (parts of) your article

$\square$ Scanning the artwork

Bibliography

If discrepancies were noted between the literature list and the text references, the following may apply:

$19 \square$ The references listed below were noted in the text but appear to be missing from your literature list. Please complete the list or remove the references from the text.

$21 \square$ Uncited references: This section comprises references that occur in the reference list but not in the body of the text. Please position each reference in the text or delete it. Any reference not dealt with will be retained inthis section

Queries and/or remarks

\begin{tabular}{|l|l|l|}
\hline $\begin{array}{l}\text { Location in } \\
\text { Article }\end{array}$ & Query / remark & Response \\
\hline AU:1 & $\begin{array}{l}\text { Has permission been obtained } \\
\text { for fig. 4 from publisher for } \\
\text { copyright? If so, please provide } \\
\text { the permission line. }\end{array}$ & \\
\hline AU:2 & $\begin{array}{l}\text { Please provide keywords for this } \\
\text { chapter. }\end{array}$ & \\
\hline AU:3 & $\begin{array}{l}\text { The ref. Zeight and Lee (1999) } \\
\text { has been changed to Leigh and } \\
\text { Zee (1999) based on the reference } \\
\text { list. Please confirm. }\end{array}$ & $\begin{array}{l}\text { The references Grönqvist et al. } \\
\text { (2006); Gredebäck et al. (in } \\
\text { prep.); Hespos et al. (submitted); } \\
\text { Baillargeon and Graber (1988) } \\
\text { are not listed in the reference list. } \\
\text { Please provide complete details } \\
\text { of the reference. }\end{array}$ \\
\hline AU:4 & \multicolumn{2}{|l}{} \\
\hline
\end{tabular}




\begin{tabular}{l|l|l|l|}
1 & AU:5 & $\begin{array}{l}\text { The year of the refs. Gibson and } \\
\text { Pick (1980); Baillargeon et al. } \\
\text { (1987) have been changed to } \\
\text { Gibson and Pick (2000); } \\
\text { Baillargeon et al. (1990) based on } \\
\text { the reference list. Please confirm. }\end{array}$ & \\
5 & & $\begin{array}{l}\text { Please provide the year of } \\
\text { publications, missing volume } \\
\text { numbers, and page ranges for } \\
\text { refs. von Hofsten et al. (in press); } \\
\text { Kochukhova and Gredebäck (in } \\
\text { press). }\end{array}$ & \\
\cline { 2 - 4 } 11 & AU:6 & &
\end{tabular}

